\title{
НЕКОДИРОВАННЫЕ ИНТЕГРИРОВАННЫЕ СИСТЕМЫ МНОЖЕСТВЕННОГО ДОСТУПА С ПЕРЕМЕЖАЮЩИМСЯ РАЗДЕЛЕНИЕМ С ИСПОЛЬЗОВАНИЕМ СТЕПЕННЫХ ПЕРЕМЕЖИТЕЛЕЙ
}

\author{
М. ЯДАВ ${ }^{1}$, В. ШОКИН ${ }^{1}$, П. К. СИНГХАЛ ${ }^{2}$ \\ ${ }^{1}$ Университет Амити, \\ Индия, Ноида \\ ${ }^{2}$ Madhav Institute of Technology and Science, \\ Индия, Гвалиор
}

\begin{abstract}
Аннотация. Перемежение (interleaving) представляет собой один из наиболее распространенных методов, предназначенных для устранения влияния многопользовательской помехи. Удаление различных помех является одной из главных задач, стоящих перед разработчиками современных систем связи. В литературе предложено несколько методов перемежения и их рабочие характеристики проанализированы в различных системах связи, доказывающих их пригодность для удаления многопользовательских помех. Одним из таких перемежителей является степенной перемежитель, который может быть использован в системе связи в качестве средства контроля пакетных ошибок и для сокращения многопользовательской помехи. В этой статье дан анализ эффективности двух различных методов интегрированного множественного доступа с перемежающимся разделением IIDMA (integrated interleave division multiple access): SCFDM-IDMA и OFDM-IDMA. Новизна этой работы состоит в том, что весь анализ выполнен с использованием степенных перемежителей для двух указанных выше методов IIDMA, поскольку традиционный метод IDMA не способен уменьшить проблемы межстанционных и межсимвольных помех. Таким образом, эти два метода при наличии степенных перемежителей делают возможной реализацию высококачественной связи без помех для систем связи будущего поколения.
\end{abstract}

Ключевые слова: перемежитель; IDMA; множественный доступ с перемежающимся разделением; степенной перемежитель; OFDM; мультиплексирование с ортогональным частотным разделением сигналов

\section{1. ВВЕДЕНИЕ}

Перемежение (interleaving) — популярный термин среди исследователей, работающих в областях беспроводной сотовой связи, цифровой связи, теории информации и/или информационной безопасности. В области связи и теории информации основная роль перемежения состоит в том, чтобы способствовать борьбе с пакетными ошибками путем распределения этих ошибок среди многих блоков данных. Таким образом, эти распределенные биты ошибки получают свою идентификацию в соответ- ствующих блоках, и поэтому могут быть обнаружены и исправлены с помощью любых кодов среднего уровня с обнаружением и исправлением ошибок [1].

Система множественного доступа с перемежающимся разделением IDMA (interleave division multiple access) представляет собой одну из современных систем связи, основанных главным образом на распределении перемежающихся шаблонов (pattern) конкретного пользователя [2]. В данном случае, пользователи различаются друг от друга в соответствии с назначенным им уникальным перемежающим- 
1. Ping, L.; Liu, L.; Wu, K. Y.; Leung, W. K. Approaching the capacity of multiple access channels using interleaved low-rate codes. IEEE Commun. Lett., v.8, n.1, p.4-6, Jan. 2004. DOI: 10.1109/LCOMM.2003.822 $\underline{534}$.

2. Ping, L.; Liu, L.; Wu, K. Y.; Leung, W. K. Interleave division multiple-access. IEEE Trans. Wireless Commun., v.5, n.4, p.938-947, Apr. 2006. DOI: 10.1109/ TWC.2006.1618943.

3. Mahadevappa, R. H.; Proakis, J. G. Mitigating multiple access interference and intersymbol interference in uncoded CDMA systems with chip-level interleaving. IEEE Trans. Wireless Commun., v.1, n.4, p.781-792, Oct. 2002. DOI: $10.1109 /$ TWC.2002.804163.

4. Kusume, K.; Bauch, G.; Utschick, W. IDMA vs. CDMA: Analysis and comparison of two multiple access schemes. IEEE Trans. Wireless Commun., v.11, n.1, p.78-87, Jan. 2012. DOI: 10.1109/TWC.2011.111211.100 954.

5. Pupeza, I.; Kavcic, A.; Ping, L. Efficient generation of interleavers for IDMA. Proc. of IEEE Int. Conf. on Communication, 11-15 June 2006, Istanbul, Turkey. IEEE, 2006, p.1508-1513. DOI: 10.1109/ICC. 2006.255024.

6. Wu, Y.-J.; Ogiwara, H. Symbol-interleaver design for turbo trellis-coded modulation. IEEE Commun. Lett., v.8, n.10, p.632-634, Oct. 2004. DOI: 10.1109/LCOMM. 2004.835320.

7. Shukla, M.; Srivastava, V. K.; Tiwari, S. Analysis and design of tree based interleaver for multiuser receivers in IDMA scheme. Proc. of 16th IEEE Int. Conf. on Networks, 12-14 Dec. 2008, New Delhi, India. IEEE, 2008, p.1-4. DOI: $10.1109 /$ ICON.2008.4772593.

8. Shukla, M.; Srivastava, V. K.; Tiwari, S. Implementation of interleavers for iterative IDMA 
receivers. Research J. Inf. Technol., v.4, n.1, p.12-21, 2012. DOI: $10.3923 /$ rjit.2012.12.21.

9. Yuan, J.; Vucetic, B.; Feng, W.; Tan, M. Design of cyclic shift interleavers for turbo codes. Annales Des Télécommunications, v.56, n.7-8, p.384-393, 2001. DOI: 10.1007/BF02995450.

10. Ren, D.; Ge, J.; Li, J. Modified collision-free interleavers for high speed turbo decoding. Wireless Personal Commun., v.68, n.3, p.939-948, 2013. DOI: 10.1007/s11277-011-0491-4.

11. Bie, H.; Bie, Z. A hybrid multiple access scheme: OFDMA-IDMA. Proc. of 1st Int. Conf. on Communications and Networking in China, 25-27 Oct. 2006, Beijing, China. IEEE, 2006, p.1-3. DOI: 10.1109/ CHINACOM.2006.344903.

12. Ping, L.; Guo, Q.; Tong, J. The OFDM-IDMA approach to wireless communication systems. IEEE Wireless Commun., v.14, n.3, p.18-24, June 2007. DOI: 10.1109/MWC.2007.386608.

13. Xiong, X.; Luo, Z. SC-FDMA-IDMA: A hybrid multiple access scheme for LTE uplink. Proc. of 7th Int. Conf. on Wireless Communications, Networking and Mobile Computing, WiCOM, 23-25 Sept. 2011, Wuhan, China. IEEE, 2011, p.1-5. DOI: 10.1109/wicom.2011. 6040400 .

14. Yadav, M.; Shokeen, V.; Singhal, P. K. BER versus BSNR analysis of conventional IDMA and OFDM-IDMA based systems with tree interleaving. Proc. of 2nd Int. Conf. on Advances in Computing, Communication, and Automation, ICACCA-Fall, 30 Sept.-1 Oct. 2016, Bareilly, India. IEEE, 2016. DOI: 10.1109/ICACCAF.2016.7748973.

15. Yadav, M.; Banerjee, P. Bit error rate analysis of various interleavers for IDMA scheme. Proc. of $3 \mathrm{rd}$ Int. Conf. on Signal Processing and Integrated Networks, SPIN, 11-12 Feb. 2016, Noida, India. IEEE, 2016, p.89-94. DOI: $\underline{10.1109 / \text { SPIN.2016.7566668. }}$.

16. Caire, G.; Guemghar, S.; Roumy, A.; Verdu, S. Maximizing the spectral efficiency of coded CDMA under successive decoding. IEEE Trans. Inf. Theory, v.50, n.1, p.152-164, Jan. 2004. DOI: 10.1109/TIT.2003.821970.

17. Wu, H.; Ping, L.; Perotti, A. User-specific chip-level interleaver design for IDMA systems. Electron. Lett., v.42, n.4, p.233-234, Feb. 2006. DOI: 10.1049/el: 20063770.

18. Ping, L.; Liu, L.; Wu, K.; Leung, W. K. Interleave-division multiple-access (IDMA) communications. Proc. of 3rd Int. Symp. on Turbo Codes and Related Topics, 2003, p.173-180.
19. Myung, H. G. Introduction to single carrier FDMA. Proc. of 15th European Signal Processing Conf., 3-7 Sept. 2007, Poznan, Poland. IEEE, 2007. URI: http://ieeexplore.ieee.org/document/7099187/.

20. Berrou, C.; Glavieux A. Near optimum error correcting coding and decoding: turbo codes. IEEE Trans. Commun., v.44, n.10, p.1261-1271, 1996. DOI: $\underline{10.1109 /}$ 26.539767.

21. Dang, J.; Zhang, W.; Yang, L.; Zhang, Z. OFDM-IDMA with user grouping. IEEE Trans. Commun., v.61, n.5, p.1947-1955, May 2013. DOI: 10.1109/TCOMM.2013.022713.120300.

22. Revathi, SP.; Juliet, A. M. Performance of OFDM IDMA system for Femtocell network. Proc. of Int. Conf. on Innovations in Information, Embedded and Communication Systems, ICIIECS, 19-20 Mar. 2015, Coimbatore, India. IEEE, 2015, p.1-4. DOI: 10.1109/ ICIIECS.2015.7193156.

23. Marne, H. D.; Mukherji, P. Comparative study of multiuser detection techniques in OFDM-IDMA systems. Proc. of Int. Conf. on Pervasive Computing, ICPC, 8-10 Jan. 2015, Pune, India. IEEE, 2015, p.1-4. DOI: 10.1109/ PERVASIVE.2015.7087209.

24. Dixit, S.; Tripathi, P.; Shukla, M. SC-FDMA-IDMA scheme for underwater acoustic communications. Proc. of Int. Conf. on Communication, Control and Intelligent Systems, CCIS, 7-8 Nov. 2015, Mathura, India. IEEE, 2015, p.204-207. DOI: $\underline{10.1109 /}$ CCIntelS.2015.7437909.

25. Dang, J.; Yang, L.; Zhang Z. Symbol detection of IDMA systems in the presence of carrier frequency offsets. Wireless Personal Commun., v.72, n.2, p.1453-1466, 2013. DOI: $10.1007 / \mathrm{s} 11277-013-1088-\mathrm{x}$.

26. Al-Iesawi, S. A. Iterative hybrid decision-feedback equalization (HDFE) based single-carrier IDMA schemes. Proc. of 6th Int. Conf. on Developments in eSystems Engineering, DeSE, 16-18 Dec. 2013, Abu Dhabi, United Arab Emirates. IEEE, 2013, p.69-72. DOI: 10.1109/DeSE.2013.21

27. Yadav, M.; Gautam, P. R.; Shokeen, V.; Singhal, P. K. Modern Fisher-Yates shuffling based optimal random interleaver design for SCFDMA-IDMA systems. Wireless Personal Commun., v.97, n.1, p.63-73, 2017. DOI: $10.1007 / \mathrm{s} 11277-017-4492-9$.

28. Yadav, M.; Shokeen, V.; Singhal, P. K. Flip left-right approach based novel inverse tree interleavers for IDMA scheme. AEU: Int. J. Electronics Commun., v.18, p.182-191, 2017. DOI: 10.1016/j.aeue.2017.07.025. 